\title{
Alterstice
}

Revue internationale de la recherche interculturelle

International Journal of Intercultural Research

Revista International de la Investigacion Intercultural

\section{Langue, culture et identité : défis et enjeux de l'intégration professionnelle des enseignants d'immigration récente en contexte francophone minoritaire}

\section{Claire Duchesne}

Volume 8, numéro 2, 2018

L'école à l'épreuve de la diversité ethnoculturelle de son personnel : regards compréhensifs croisés

URI : https://id.erudit.org/iderudit/1066949ar

DOI : https://doi.org/10.7202/1066949ar

Aller au sommaire du numéro

Éditeur(s)

Alterstice

ISSN

1923-919X (numérique)

Découvrir la revue

Citer cet article

Duchesne, C. (2018). Langue, culture et identité : défis et enjeux de l'intégration professionnelle des enseignants d'immigration récente en contexte francophone minoritaire. Alterstice, 8(2), 13-24.

https://doi.org/10.7202/1066949ar

\section{Résumé de l'article}

Lorsqu'ils ont trouvé un poste d'enseignant dans les écoles de l'Ontario français, les nouveaux enseignants issus de l'immigration font face au défi de s'intégrer pleinement au corps enseignant dont ils font nouvellement partie, entre autres par l'appropriation de leur rôle de passeur culturel à l'égard de la construction identitaire de leurs élèves, de même que par le processus de (re)construction de leur propre identité en tant qu'enseignants francophones oeuvrant en contexte minoritaire. En s'appuyant sur ses recherches antérieures de même que sur les écrits qu'elle a recensés, l'auteure vise une meilleure compréhension des défis et des enjeux relevant de l'intégration professionnelle des nouveaux enseignants issus de l'immigration, particulièrement sur les plans des enjeux contextuels et identitaires qui sont inhérents au travail en situation francophone minoritaire. 


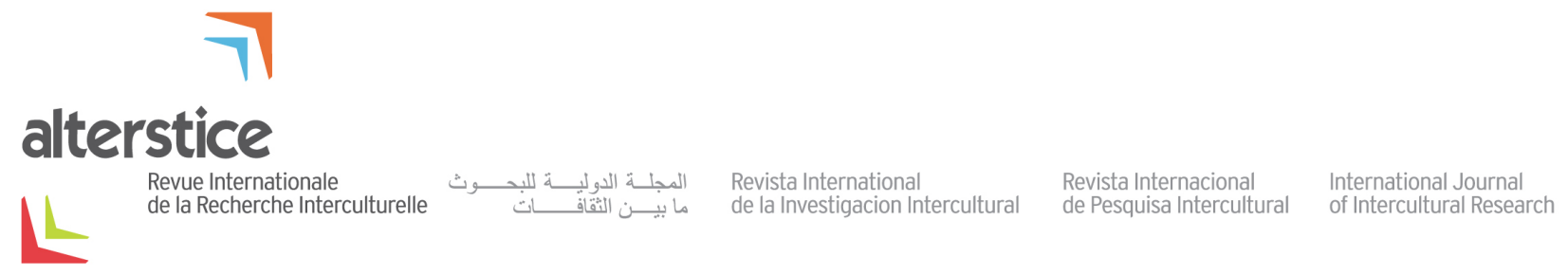

ARTICLE THÉMATIQUE

\section{Langue, culture et identité : défis et enjeux de l'intégration professionnelle des enseignants d'immigration récente en contexte francophone minoritaire}

Claire Duchesne $^{1}$

\section{Résumé}

Lorsqu'ils ont trouvé un poste d'enseignant dans les écoles de l'Ontario français, les nouveaux enseignants issus de l'immigration font face au défi de s'intégrer pleinement au corps enseignant dont ils font nouvellement partie, entre autres par l'appropriation de leur rôle de passeur culturel à l'égard de la construction identitaire de leurs élèves, de même que par le processus de (re)construction de leur propre identité en tant qu'enseignants francophones œuvrant en contexte minoritaire. En s'appuyant sur ses recherches antérieures de même que sur les écrits qu'elle a recensés, l'auteure vise une meilleure compréhension des défis et des enjeux relevant de l'intégration professionnelle des nouveaux enseignants issus de l'immigration, particulièrement sur les plans des enjeux contextuels et identitaires qui sont inhérents au travail en situation francophone minoritaire.

\section{Rattachement de l'auteure \\ ${ }^{1}$ Université d'Ottawa, Ottawa, Canada \\ Correspondance}

claire.duchesne@uottawa.ca

\section{Mots clés}

Intégration professionnelle, enseignants issus de l’immigration, francophonie minoritaire, Ontario français

\section{Pour citer cet article}

Duchesne, C. (2018). Langue, culture et identité : défis et enjeux de l'intégration professionnelle des enseignants d'immigration récente en contexte francophone minoritaire. Alterstice, 8(2), 13-24. 


\section{Introduction et problématique}

En ces temps de pénurie d'enseignants francophones, les écoles de l'Ontario français accueillent un nombre croissant de nouveaux enseignants issus de l'immigration (Lefebvre, 2017 ; Murphy, 2018 ; Pierroz, 2018). Bien que considérés "nouveaux» dans la profession au Canada, certains d'entre eux (mais pas tous) ont connu des expériences d'enseignement dans leur pays d'origine ou dans un pays de transition. Ces néo-Canadiens ont, la plupart du temps, quitté des régions où sévissait l'instabilité politique, afin de procurer à leurs enfants un milieu de vie dans lequel ces derniers pourraient grandir, s'instruire et vivre en toute sécurité (Sinacore, Park-Saltzman, Mikhail et Wada, 2010). Ainsi, depuis une vingtaine d'années maintenant, le programme francophone de formation à l'enseignement de la Faculté d'éducation de l'Université d'Ottawa a vu ses cohortes d'étudiants s'enrichir de ressortissants principalement originaires d'Afrique subsaharienne et du Maghreb. Bon an mal an, ces étudiants issus de l'immigration forment environ $40 \%$ de la population étudiante totale des campus d'Ottawa, de Toronto et de Windsor et, par conséquent, constituent une entité importante des centaines de diplômés qui, chaque année, tentent de trouver une place au sein des 12 conseils scolaires francophones de l'Ontario (qui regroupent plus de 400 écoles) ou dans les organisations scolaires des autres provinces et territoires canadiens.

Mes tâches professorales au sein de ce programme de formation à l'enseignement m'ont rapidement fait prendre conscience des défis rencontrés par les étudiants issus de l'immigration, tant dans leur socialisation au métier d'étudiant dans une université nord-américaine que dans leurs fonctions de stagiaires dans les écoles de l'Ontario français. Au fil des divers projets de recherche que j'ai menés et des années à côtoyer ces étudiants dans mes classes, j'ai eu l'occasion, entre autres, d'étudier plus en profondeur les conceptions de l'enseignement des futurs enseignants issus de l'immigration que nous formons, de même que les enjeux associés à leur insertion professionnelle. Outre mes préoccupations pour l'avenir des communautés francophones hors Québec, mes réflexions actuelles sur le sujet m'ont incitée à $m^{\prime}$ 'interroger sur le rôle et l'expérience des enseignants d'immigration récente dans la profession enseignante, et ce, dans le cadre très particulier des communautés francophones en contexte minoritaire. Quelle est cette francophonie canadienne vivant en milieu minoritaire? Quels sont les rôles de l'enseignant francophone, notamment en Ontario ? Quels sont les défis rencontrés par les enseignants issus de l'immigration dans ce contexte ? Ces questions, parmi d'autres, ont alimenté les réflexions qui ont conduit à la rédaction de cet article. Ainsi, à partir de l'analyse des enjeux contextuels et identitaires soulevés par ce sujet, ce texte fait état de l'expérience d'intégration à la profession enseignante des enseignants d'immigration récente en milieu scolaire francophone de l’Ontario.

\section{Contextualisation : francophonie québécoise et hors Québec}

Quand on est né au Québec et que l’on y a résidé toute sa vie, comme c'est mon cas, on est souvent peu informé des défis que doivent relever ceux qui vivent en contexte linguistique minoritaire. La présence francophone à l'extérieur du Québec, bien qu'elle soit mal connue, n'est pourtant pas nouvelle. Assurée par les Français et autres Européens de souche francophone aux temps de la colonisation, puis par les Québécois en quête d'emploi dans l'industrie ou à la recherche de terres à cultiver, c'est maintenant à l'arrivée d'immigrants francophones désireux de s'établir et de contribuer à la société canadienne que cette francophonie doit sa vitalité (Harvey, 1995 ; Lalonde, 1995 ; Sylvestre, 2013).

Pour leur part, quand ils se comparent à l'anglophonie qui les entoure, les Québécois de langue française se savent minoritaires pour ce qui est du nombre de locuteurs. À l'intérieur des frontières de la province, cependant, ils vivent en situation majoritaire, surtout en dehors des régions de Montréal, de l'Estrie et de l'Outaouais où se concentrent les communautés anglophones du Québec. Qui plus est, le Québec a tellement bien réussi à se positionner en tant que territoire francophone sur l'échiquier mondial qu'il s'y est plus ou moins installé comme lieu "ultime» de la francophonie canadienne, poussant alors dans l'ombre l' «autre » francophonie du pays. Comme le mentionne Fourot (2016), ces francophonies, québécoise et hors Québec, ont évolué en rivalité, le Québec constituant le déterminant de leurs identités respectives : « [a]insi, la francophonie minoritaire canadienne était comprise/envisagée en opposition/rivalité à la francophonie majoritaire québécoise » (p. 30). Conséquemment, les francophones de l'ensemble du pays ont dès lors été désignés comme ceux vivant «à l'extérieur » de cette province, alors que les francophones reconnus par le gouvernement fédéral comme « nation 
distincte ${ }^{1}$ vivent " à l'intérieur » du Québec. Pourtant, comme l'affirmait déjà Cardinal (1994) il y a plus de 20 ans, «[...] il est vain de toujours s'en prendre [au Québec], de lui reprocher son abandon des francophones et de se cacher le rôle de l'État fédéral dans ce mouvement » (p. 77). En somme, bien qu'il soit reproché au Québec d'avoir fait peu de cas des luttes qu'ont mené les francophones des autres provinces pour la revendication de leurs droits, il demeure que l'harmonisation de la reconnaissance des langues officielles et des droits des minorités linguistiques entre les provinces relève de l'appareil fédéral.

D’un point de vue historique, les Québécois de langue française constituent le groupe majoritaire de leur province et, jusqu'aux années 1990, les anglophones formaient le principal groupe minoritaire sur lequel reposait ce rapport d’opposition. Le paysage démographique du Québec s'est toutefois grandement modifié depuis les années 2000. En effet, à propos du rapport de dualité qui s'est installé à la suite de l'arrivée massive d'immigrants dans la communauté francophone canadienne, notamment au Québec, Létourneau et Gani (2017) postulent que « [I]e fait de distinguer, à l'intérieur de la nation québécoise, ceux que l'on identifie exclusivement comme "Québécois d'origine canadienne-française" et ceux que, dans les circonstances spécifiques de ce texte, on qualifiera d' "autres Québécois" n'est évidemment pas sans poser problème »(p. 7). Les immigrants sont ainsi devenus la nouvelle entité minoritaire, et ce, à l'intérieur même de la francophonie canadienne, que celle-ci soit majoritaire, comme au Québec, ou minoritaire, comme dans les autres provinces et territoires du pays.

En ce qui me concerne, c'est en étudiant d'abord, puis en travaillant à l'Université d'Ottawa, une université bilingue ayant pour mission première de servir la population francophone de l'Ontario, que j'ai pris conscience que, des 7,3 millions de citoyens canadiens, natifs et issus de l'immigration, ayant le français comme langue maternelle (soit $22 \%$ de la population totale du pays), 1 million d'entre eux se trouvaient en dehors du Québec. De ce nombre de francophones vivant en contexte minoritaire, $56 \%$ résident en Ontario et $24 \%$ au Nouveau-Brunswick, les autres étant essentiellement établis dans les provinces de l'Ouest (Statistique Canada, 2011). Dans les faits, comme le souligne Cardinal (1994), il n'y pas qu'une seule francophonie canadienne, mais plusieurs: "[I]es francophones habitent des îles différentes, avec des populations aux accents mélangés, aux identités multiples, aux caractéristiques rurales ou urbaines, aux racines d'ici ou d'ailleurs " (p. 7). Ainsi, à ces communautés francoontarienne, acadienne, fransaskoise et autres francophonies canadiennes, s'ajoutent désormais des francophonies internationales provenant notamment du continent africain.

\section{De nouveaux enseignants issus de l'immigration}

La pénurie d'enseignants causée par les départs massifs à la retraite des baby-boomers dans les années 2000 s'est à peu près résorbée au tournant de la décennie suivante. Dès lors, afin de diminuer le nombre de nouveaux enseignants affluant dans les écoles et pour répondre aux pressions intra- et interprovinciales de bonifier les programmes de formation à l'enseignement offerts dans les 12 universités de la province (dont deux programmes en français : Ottawa et Laurentienne), le gouvernement de l'Ontario a autorisé la prolongation (d'une année à deux années) de la durée de ces programmes, sans toutefois permettre l'augmentation du nombre d'admissions. Ainsi, depuis 2016, l'Ontario n'assure désormais la diplomation annuelle que de la moitié de ses aspirants-maitres puisque le nombre total des admis, qui est demeuré le même, se répartit maintenant sur un cycle biennal. Cette diminution radicale des diplômés en enseignement s'est traduite par une nouvelle pénurie d'enseignants, de façon cependant plus marquée du côté francophone (Ordre des enseignantes et des enseignants de l'Ontario [OEEO], 2017 ; Pierroz, 2018). Bien que cet ajout d'une année d'études ait eu pour conséquence de refroidir l'intérêt d'un nombre important de postulants, le programme francophone de formation à l'enseignement de l'Université d'Ottawa accueille toujours la même proportion de candidats d'immigration récente qui, devant la difficulté de trouver un emploi dans les domaines pour lesquels ils étaient qualifiés avant la migration, choisissent la profession enseignante pour assurer leur intégration à leur société d'accueil. Pourtant, malgré la pénurie observée, " [I]a moitié (49\%) des néo-Canadiens certifiés en Ontario ne trouve pas de travail dans sa première année de carrière ॥ (OEEO, 2017, p. 33), ce qui constitue le groupe d'enseignants ayant le plus de difficulté à trouver un emploi.

\footnotetext{
${ }^{1}$ Le 28 novembre 2006, Radio Canada informait que le Parlement canadien avait adopté «la motion du premier ministre Stephen Harper, qui reconnaît que les Québécois forment une nation au sein d'un Canada uni ». https://ici.radiocanada.ca/nouvelle/331287/vote-nation
} 
Paradoxalement, la présence des nouveaux enseignants issus de l'immigration (dorénavant NEII) est grandement souhaitée dans les écoles de l'Ontario, afin d'assurer une certaine représentativité auprès de la population scolaire de plus en plus diversifiée de cette province. Déjà, en 1994, la Commission royale sur l'éducation, mandatée par le gouvernement de l'Ontario, recommandait (Article 61) :

[...] que les facultés d'éducation augmentent leurs efforts pour admettre plus d'étudiantes-enseignantes et d'étudiantsenseignants provenant de groupes précédemment sous-représentés, notamment les minorités ethnoculturelles et raciales, les groupes autochtones et les personnes handicapées, et qu'elles soient responsables de montrer à l'Ordre des enseignantes et des enseignants que des progrès notables sont réalisés dans l'atteinte de cet objectif.

Même lorsqu'ils sont munis d'un diplôme universitaire canadien et de l'accréditation de l'Ordre des enseignantes et des enseignants de l'Ontario, ces NEII rencontrent tout de même plus de difficultés à s'insérer dans la profession que leurs collègues canadiens natifs. Des constats similaires ont également été réalisés dans les écoles francophones albertaines (Mulatris et Skogen, 2012) et québécoises (Niyubahwe, Mukamurera et Jutras, 2014).

\section{Quelques précisions à propos de l'insertion et de l'intégration professionnelles}

Il importe de clarifier et de distinguer deux concepts importants qui fournissent un cadre de référence aux propos qui sont tenus dans cet article, soit celui d'insertion professionnelle et celui d'intégration professionnelle. Je m'appuierai pour cela sur les travaux d'Huberman (1989) : ce chercheur suisse et son équipe ont consacré cinq ans de travail à comprendre et à décrire le cycle de vie professionnelle des enseignants du secondaire. Sept phases caractérisant le processus de développement professionnel de la majorité des 160 enseignants, hommes et femmes, interviewés lors de cette étude, ont été identifiées. Je m’intéresse ici particulièrement aux deux premières phases du cycle, soit celles d'exploration et de stabilisation, qui se rapportent respectivement, à mon sens, aux processus d'insertion puis d'intégration dans la profession enseignante.

L'insertion professionnelle en enseignement, correspondant à la phase d'exploration (de 1 à 3 ans d'expérience ${ }^{2}$ ), selon Huberman (1989), renvoie au stade de la survie et à celui de la découverte. Lors de la période de survie, le nouvel enseignant fait face à la complexité de la tâche, au tâtonnement, aux doutes sur sa compétence et à l'apprentissage de tous les aspects de la profession, allant des contenus disciplinaires à la gestion de classe. La découverte, quant à elle, est source d'enthousiasme pour le novice puisqu'elle lui permet d'expérimenter, d'explorer, de faire partie d'une profession définie, d'avoir sa classe à soi et d'assumer l'entière responsabilité de l'enseignement à un groupe spécifique d'élèves. Les stades de survie et de découverte s'exercent simultanément, le second compensant pour l'anxiété générée par le premier. La phase d'exploration (ou d'insertion professionnelle dans le cas de cet article), selon les expériences qui y seront vécues et les aspirations de l'enseignant novice, conduira ce dernier soit vers la phase suivante, la stabilisation, soit vers la décision de quitter la profession.

L'intégration professionnelle en enseignement qui, selon Huberman (1989), correspond à la phase de stabilisation (de 4 à 6 ans d'expérience), se caractérise par le choix de s'engager définitivement dans la carrière enseignante. La difficulté inhérente à cette étape réside justement dans l'obligation de faire un choix : l'individu, en optant pour l'enseignement, choisit une identité professionnelle précise et renonce ainsi à d'autres identités potentielles comme à d'autres possibilités de carrière. Lors de la phase de stabilisation, l'enseignant s'affirme davantage devant ses collègues et ses supérieurs, s'émancipe et expérimente différents degrés de liberté professionnelle. Sur le plan pédagogique, l'enseignant s'achemine vers l'acquisition du sentiment de maîtrise didactique et celui de compétence professionnelle. Il est moins centré sur sa propre personne et, même s'il ne sait pas encore faire face à toutes les situations, il n'en demeure pas moins un intervenant efficace. D'après l'auteur, la phase de stabilisation (ou d'intégration à la profession dans cet article) constitue, habituellement, une étape vécue agréablement et positivement par l'enseignant.

\footnotetext{
${ }^{2}$ D'après certaines recherches récentes sur l'insertion professionnelle des enseignants et selon les contextes particuliers aux différentes organisations scolaires, des enseignants canadiens peuvent vivre une phase d'insertion professionnelle ou d'exploration pouvant s'étaler sur une période allant jusqu'à 5 ou 7 ans.
} 
Dans le cas des NEII dont il est question dans cet article, la phase de stabilisation (ou d'intégration professionnelle) correspond à la période au cours de laquelle se construit l'identité professionnelle de l'enseignant, mais également l'identité de passeur culturel, lorsqu'il enseigne en contexte de francophonie minoritaire.

\section{Les défis du contexte minoritaire pour les NEII de l'Ontario français}

Les difficultés associées à l'insertion professionnelle des nouveaux enseignants de manière générale ont été largement décrites dans la documentation savante, mais nous possédons relativement peu d'informations en ce qui concerne cette expérience pour les enseignants issus de l'immigration, qu'ils soient nouveaux ou non dans la profession. La recension de Niyubahwe, Mukamurera et Jutras (2013) a permis d'établir quatre types de difficultés liées à l'insertion professionnelle de ces derniers en Amérique du Nord, en Australie et en Israël, soit les difficultés relatives 1) à l'embauche, 2) à la culture éducative et à l'équipe enseignante, 3) à la non-reconnaissance des compétences et des qualifications provenant du pays d'origine et 4) à la gestion de l'enseignement de même qu'à celle de la classe. Pour leur part, les travaux de Morrissette, Charara, Boily et Diédhiou (2016) et de Morrissette et Demazière (2018) ont mis en relief les particularités de la socialisation au métier et au groupe professionnel des enseignants d'immigration récente des écoles montréalaises. Les NEll de l'Ontario français auxquels je m'intéresse depuis plus d'une dizaine d'années, quant à eux, ont rencontré des défis similaires à ceux précédemment énoncés (Duchesne, 2008, 2010a, 2010b et 2017b), mais également des difficultés relevant des perspectives racialisées dont ils font l'objet (Duchesne, soumis) et du contexte très particulier que constitue l'enseignement en milieu minoritaire francophone. Comme il le sera exposé dans les prochaines sections, les écrits que j'ai recensés et les différents témoignages que j'ai recueillis lors d'études antérieures portant, d'une part, sur les conceptions de l'enseignement et de l'apprentissage des NEIl et, d'autre part, sur leur insertion professionnelle dans les écoles de I'Ontario français, mettent en relief deux problématiques, étroitement liées, qui se posent à ces enseignants au terme de la phase d'insertion professionnelle. En effet, il semble que l'appropriation de leur rôle de passeur culturel à l'égard de la construction identitaire des élèves en milieu francophone minoritaire de même que le processus de (re)construction identitaire auquel ils doivent eux-mêmes faire face constituent des défis contextuels rencontrés par les NEIl de la francophonie ontarienne au moment de leur intégration à leur nouvelle communauté professionnelle qu'il importe de prendre en considération.

\section{Les NEll en tant que passeurs culturels}

Le flux migratoire que connaît le Canada contribue à la croissance de ses communautés francophones en contexte minoritaire et, du même coup, à la redéfinition de l'école francophone. D'après Cavanagh, Cammarata et Blain (2016) :

Comme elle fait partie intégrante de cette réalité, l'école francophone ne peut plus être considérée comme une école homogène sur les plans linguistique, culturel et identitaire. En effet, depuis quelques années, surtout dans les grands centres urbains, le rythme d'arrivée des immigrants s'est accéléré et leur diversité a grandi. La clientèle francophone comprend maintenant des élèves francodominants, anglodominants et plurilingues. (p.6)

Gérin-Lajoie (2006), pour sa part, souligne que les écoles françaises de l'Ontario ne sont pas des milieux d'immersion visant à développer les compétences linguistiques des élèves dont la langue d'usage est l'anglais, mais des écoles où le français est la langue première d'enseignement et où l'anglais constitue une matière obligatoire, certes, mais secondaire. L'auteure signale également certaines particularités de la population étudiante de ces écoles qui :

[...] est souvent diversifiée en matière de compétences langagières en français. Plusieurs élèves parlent en effet couramment le français, alors que d'autres parlent peu et parfois même pas du tout cette langue, au moment où ils et elles arrivent à l'école. Même les élèves qui parlent le français couramment préfèrent souvent l'anglais au français. (p. 165)

Qui plus est, les représentations identitaires évoluent et, d'après Sylvestre et Lévesque (2018), "un très grand nombre de jeunes Franco-Ontariens se définissent désormais comme "bilingues francophones" ॥ (p. 32), ce qui complexifie la mission des écoles de langue française. La forte immigration vers l'Ontario ajoute une dimension interculturelle aux enjeux linguistiques: Gérin-Lajoie (2018) constate, à cet effet, que "l'avènement d'une 
population scolaire diversifiée où langues et cultures s'entrecroisent a contribué à une hétérogénéité linguistique et culturelle encore plus grande » (p. 41).

Afin de soutenir la construction identitaire des élèves francophones et francophiles de la province, le ministère de l'Éducation de l'Ontario (MÉO) a mis en œuvre la Politique d'aménagement linguistique (PAL), dont la mission consiste à :

préserver la profonde originalité de la culture de la communauté francophone de l'Ontario et [à] assurer le renouvellement de son expression dans le temps et l'espace, en particulier par les apports de populations de langue française de toutes origines et par l'exploitation du vaste fonds de références culturelles ainsi que des nombreux réseaux sociaux, professionnels et culturels de la francophonie. (MÉO, 2011, p. 1)

En outre, dans le rapport qu'il produisait, 10 ans après sa création, à la suite d'une consultation provinciale au sujet de la PAL, le MÉO (2014) précisait :

Afin d'offrir un enseignement de qualité adapté à la situation minoritaire, les membres du personnel enseignant doivent maîtriser la langue française et posséder une solide formation en matière de pédagogie culturelle, de communication orale, d'actualisation en langue française et en diversité culturelle. (p. 12)

Pour mener à bien cette initiative, le ministère compte par ailleurs sur l'engagement d'enseignants " ayant la capacité à œuvrer en milieu minoritaire » (p. 12). Cette capacité se fonde, entre autres, sur une formation continue incluant des modules destinés aux nouveaux enseignants qui ne sont pas issus de la francophonie minoritaire ontarienne, dont les NEIl. Le rapport de 2014 indique également que "[s]elon de nombreux répondants, ces nouvelles enseignantes et nouveaux enseignants doivent être tenus de contribuer à la construction identitaire des élèves au même titre que les enseignantes et les enseignants issus de milieux francophones en Ontario » (p. 13). Dès lors, le rôle de l'enseignant en contexte francophone minoritaire dépasse les frontières de la pédagogie en s'inscrivant dans une démarche politique, ce qui a pour conséquence d'ajouter aux nombreux défis que doivent relever les NEII qui enseignent dans ce contexte. Comme le souligne Gérin-Lajoie (2018), à propos de l'école de langue française en Ontario : " [m]ême si le présent discours officiel n'y fait pas directement référence, il n'en demeure pas moins que cette institution a toujours été, et continue de l'être, un élément indispensable dans les luttes pour les droits des francophones et la survie de la langue et de la culture d'expression française » (p. 51).

Ainsi, pour un NEII dont le français constitue la $2^{\mathrm{e}}$ ou la $3^{\mathrm{e}}$ langue d'usage et pour qui cette langue est associée à un passé plus ou moins marqué par la colonisation, l'appropriation du rôle d'agent de reproduction linguistique et, plus encore, l'identification au rôle de passeur de la culture francophone peuvent présenter des défis d'importance. Comme le souligne Mulatris à propos des immigrants africains (2009) :

Le positionnement politique par rapport à la langue française est une réalité nouvelle pour la plupart de ceux-ci. Leur contexte d'origine ne les a pas préparés aux enjeux de la francophonie en situation minoritaire. Langue de la colonisation, le français a toujours été, pour la plupart de ces immigrants africains, la langue de l'élite et du pouvoir. L'arrivée au Canada impose une démarche d'apprentissage de l'histoire, des revendications en matière des droits et du partage des ressources publiques. Cet apprentissage est, en un sens, nécessaire si l'immigrant veut développer un sens d'appartenance à la communauté d'accueil, mais il exige des efforts de redéfinition des identités francophones minoritaires. (p. 149)

En effet, parmi les NEll que j'ai côtoyés dans mes classes à la Faculté d'éducation comme au cours de mes recherches, nombre d'entre eux ne portaient pas, d'emblée, le drapeau de l'identité francophone et demeuraient perplexes devant le rôle de premier plan qu'ils auraient ou avaient à jouer pour soutenir la construction identitaire de leurs élèves. Un directeur adjoint ayant participé à mes travaux souligne, d'ailleurs, le poids que cette exigence revêt pour les NEIl par rapport aux enseignants natifs: "On va leur demander, en plus de cela, de faire de la construction identitaire et pas nécessairement [celle de] leur culture [...] je trouve qu'on leur en demande encore plus $[\ldots] »$.

Pour ces NEII, c'est d'abord leur appartenance à un groupe ethnoculturel particulier et, dans plusieurs cas, leur statut de minorité visible qui détermine, à leurs yeux, leur situation de minoritaires. En immigrant au Canada, ils 
n'avaient pas prévu que la langue française, pourtant relativement bien maitrisée par la majorité d'entre eux, constituerait un enjeu politique et, dans plusieurs cas, une difficulté de plus à leur intégration sociale et professionnelle. Comme le souligne Fourot (2016) :

Les difficultés rencontrées par les immigrants francophones sont en de nombreux points similaires à celles des immigrants en général. Toutefois, leur statut de minorité au sein d'une minorité constitue un obstacle supplémentaire, en particulier pour les minorités racialisées. (p. 39)

Dans un contexte ou l'anglais constitue la langue de la majorité, Jacquet, Moore et Sabatier (2008) voient, dans cette combinaison identitaire des statuts de francophone, d'immigrant et de minorité visible, une «triple minorisation » (p. 92) accentuant l'expérience discriminatoire vécue. Plus encore, certains des NEII qui ont témoigné de leur expérience lors de l'une de mes recherches ne soupçonnaient pas que leur rôle de passeur culturel auprès des élèves évoluant en contexte minoritaire puisse avoir de possibles répercussions sur leurs façons d'enseigner, de même que sur le regard que poseraient sur eux leurs collègues enseignants et leurs dirigeants scolaires (Duchesne, 2017a). J'ai en effet constaté, lors d'entretiens que j'ai menés auprès de NEll, qu'ils exprimaient peu, voire pas du tout, leurs préoccupations à propos de la construction identitaire de leurs élèves ou des enjeux auxquels faisait face la francophonie en contexte minoritaire. Au moment de leur insertion professionnelle, et malgré qu'ils aient été sensibilisés, lors de leur passage à la Faculté d’éducation, à cette réalité, ils n'étaient peut-être pas conscients, voire peu ou mal informés, des responsabilités qui leur incombaient à cet égard. Comme l'a mentionné une NEll originaire du Maghreb: " mais c'est quoi, au juste, l'identité francoontarienne? ».

Pour leur part, aux yeux de la communauté francophone ontarienne, les dirigeants scolaires sont responsables d'assurer la mise en place d'interventions soutenant la construction identitaire des élèves de leur école. Certains de ceux que nous avons rencontrés ont exprimé leur inquiétude en ce qui concerne la compréhension et l'appropriation du rôle de passeur culturel en contexte minoritaire que doivent endosser les NEII. En effet, des directeurs ont mentionné que pour les NEll qui ont reçu une éducation héritée du colonialisme français, les exigences envers leurs élèves, en matière de maitrise linguistique à l'oral comme à l'écrit, dépassaient ce qu'il convenait d'attendre de la part d'enfants et d'adolescents qui vivent dans des milieux fortement influencés par la langue anglaise. Comme l'a mentionné un dirigeant scolaire ayant participé à mes travaux : "l'anglais, en Ontario, on ne l'apprend pas, on l'attrape "! Les interventions des enseignants, en ce sens, doivent favoriser la fierté d'être francophone, l'intérêt des élèves pour la langue française et le désir d'en maintenir la vitalité ; ces interventions doivent, par conséquent, éviter de décourager les efforts des élèves ou, pire encore, de susciter en eux des sentiments d'insécurité et d'incompétence linguistiques, ce qui est souvent le lot des francophones évoluant en contexte minoritaire (Cavanagh, Cammarata et Blain, 2016).

\section{De l'insécurité culturelle et linguistique à la (re)construction identitaire}

Il est reconnu par les instances gouvernementales que la survie de la francophonie canadienne en contexte minoritaire passe par l'intégration des immigrants de langue française à ses communautés, et des initiatives sont mises de l'avant afin de favoriser l'immigration de langue française à cet effet (Gouvernement du Canada, 2018). L'Ontario, qui est la province canadienne recevant le plus grand nombre de nouveaux arrivants, voit ainsi ses écoles francophones accueillir une population étudiante de plus en plus diversifiée. Bien que la diversification du corps enseignant soit également souhaitée pour assurer une meilleure représentativité auprès des élèves, la question du clivage "Eux/Nous » se pose chez le personnel enseignant des écoles de l'Ontario français, freinant du même coup les tentatives de socialisation de ses membres, natifs et non-natifs, nécessaires à leur unification.

Ainsi, l'insécurité vécue par les personnes qui appartiennent à un groupe minoritaire peut amener celles-ci à ressentir l'arrivée ou la présence d'un autre groupe minoritaire comme une menace à sa sécurité, voire à son identité (Sinacore et collab., 2010). Chaque groupe minoritaire poursuit ses propres luttes pour faire valoir ses droits devant le groupe majoritaire ; dès lors, pour protéger ses acquis et apaiser son sentiment d'insécurité, il est susceptible de mettre ses rivaux au défi. 
Les rivalités dominants/dominés entre francophones, au Canada, ne se fondent pas uniquement sur les déterminants géographiques Québec/hors Québec, mais également sur des marqueurs identitaires relevant du bagage génétique et ethnoculturel des membres de ces groupes. D’un côté, les francophones considérés « de souche ", "de sang ", ou "généalogiques " dont les ancêtres sont les colonisateurs venus d'Europe de l'Ouest et, de l'autre, les immigrants de $1^{\mathrm{e}}$ ou $2^{\mathrm{e}}$ génération, les " minorités visibles » ou "les ethniques ", considérés comme étrangers par ceux du premier groupe. Dès lors, ces « ruptures et fragmentations de l'identité francophone en milieu minoritaire " (Cardinal, 1994, p. 71), engendrent la minorisation des enseignants issus de l'immigration à l'intérieur même de la minorité linguistique. Ainsi, deux groupes minoritaires en quête de reconnaissance identitaire affaiblissent leur position devant le groupe majoritaire de langue anglaise, en nourrissant une opposition qui conduit inévitablement à la division, plutôt qu'en se solidarisant. Comme le mentionne Fourot (2016) :

les relations majoritaire/minoritaire ne sont pas figées une fois pour toutes et un minoritaire peut se comporter en majoritaire dans d'autres relations de pouvoir. [...] Ce qui caractérise un groupe minoritaire, c'est la relation qu'il entretient avec les autres groupes: en fait, ce sont les rapports de domination qui caractérisent les groupes minoritaires, plus que la marque qui les identifie de manière arbitraire. (p. 31)

Dans cette optique, l'insécurité qui est à la source de la rivalité manifestée par ces deux groupes d'enseignants qui évoluent en contexte minoritaire, l'un « de souche » et l'autre racialisé, pourrait se fonder sur la question de savoir quelle(s) culture(s), et provenant de quelle francophonie, sera/seront privilégiée(s) dans le milieu de travail et d'éducation que constitue l'école.

\section{De la construction identitaire...}

Les écrits de Dubar (2010) apportent un éclairage judicieux sur le processus de construction identitaire. L'auteur précise, à propos de la construction de l'identité sociale, que «[c]elle-ci n'est pas choisie, mais conférée par les institutions et les proches non seulement sur la base des appartenances ethniques, politiques, religieuses, professionnelles et culturelles de ses parents, mais aussi sur celles de ses performances scolaires » (p. 112). Ainsi, en s'appuyant sur les bases élaborées dès l'enfance, l'individu poursuivra la construction de son identité sociale, à l'âge adulte, par le choix d'un domaine professionnel. En ce qui concerne les enseignants des écoles en milieu linguistique minoritaire, cette identité, point marquant de la phase d'intégration à la profession, sera consolidée par la poursuite d'une mission commune relevant de la perpétuation de la langue et de la culture afin d'assurer l'épanouissement des membres de la communauté qu'elle sert. Ces préoccupations demeurent fondamentales car, comme le soulignent Lévesque, Croteau et Gani (2015) :

si l'on souhaite "l'appropriation par l'élève de la culture francophone », comme l'exige le programme-cadre ontarien, il nous parait donc important de s'intéresser sérieusement aux visions historiques des jeunes, ainsi qu'à leur sentiment d'appartenance envers la communauté. Car apprendre et comprendre, comme le rappelle Henri Moniot, «c'est relier du nouveau à ce que l'on sait déjà, à ce que l'on croit déjà, à ce qu'on est... » (p. 221)

Ces « visions historiques » s'appuyant sur " ce que l'on sait déjà, ce que l'on croit déjà » et " ce qu'on est » revêtent des nuances différentes selon les expériences vécues, mais surtout, dans le cas qui nous concerne, selon les pays d'où l'on vient, le patrimoine qui nous a été légué et la culture dans laquelle on a évolué. De plus, si les enjeux associés à la construction identitaire des élèves de l'Ontario français semblent assez bien circonscrits en matière linguistique, ils le sont beaucoup moins sur le plan culturel. En effet, le rôle de passeur culturel des enseignants de ces écoles demeure flou: compte tenu des flux migratoires qui transforment le paysage démographique de I'Ontario, la présence des NEII dans les écoles peut inciter la communauté scolaire à poser ces questions : quelle culture sera transmise? (la culture ethnicisée du NEIl ou celle des Franco-Canadiens telle que le NEII se la représente ?) et quelle culture devrait être transmise? (une culture définie par le ministère de l'Éducation, une culture canadienne exempte de toute régionalisation ou une culture représentative de la diversité francophone, ethnique et sociétale ?) en somme, de quelle(s) culture(s) est-il ici question ?

Alterstice - Revue Internationale de la Recherche Interculturelle, vol. $8, n^{\circ} 2$ 
...à la reconstruction de l'identité...

Par ailleurs, afin de s'approprier pleinement le rôle de passeur culturel dans les écoles de l'Ontario français, il est nécessaire que les enseignants soient pleinement conscients des enjeux associés à l'éducation en contexte minoritaire. Il est aussi implicitement attendu qu'ils redéfinissent leur identité en conséquence, puisqu'il ne s'agit pas simplement pour eux de jouer un rôle, comme on incarne un personnage au théâtre pendant quelques heures ou quelques semaines, mais d'un statut à intégrer à leur registre identitaire pour la durée de leur carrière. Le rôle de passeur culturel visant à soutenir la construction de l'identité francophone des élèves invite l'enseignant à reconstruire sa propre identité. En effet, pour ces enseignants qui poursuivent leur parcours de vie lors d'une transition vers l'enseignement en milieu francophone minoritaire, "[I]es multiples tensions que vit l'individu dans les périodes de transition professionnelle génèrent des transformations plus ou moins profondes au plan identitaire, engageant un processus de déconstruction/reconstruction qui accompagne le remaniement du rapport entre soi et l'environnement " (Balleux et Perez-Roux, 2013, p. 107). Ainsi le NEll qui souhaite intégrer pleinement le corps enseignant œuvrant dans les écoles francophones de l'Ontario sera appelé à se situer et sans doute à se repositionner en tant qu'immigrant, en tant que possible membre d'une minorité visible, en tant que néoCanadien, en tant que francophone et agent de reproduction linguistique et en tant qu'enseignant en contexte minoritaire. Ces constats font écho à ceux de Madibbo (2009-2010) à propos des multiples formes d'appartenance ressenties par les immigrants dont la chercheure a recueilli les témoignages :

Notre analyse indique que les immigrés insistent sur leur volonté de s'identifier à la société d'accueil. De plus, comme nous le rappelle un participant à notre recherche qui signale «tout ceci [identification à la communauté d'origine, à la francophonie et au Canada] fait partie de moi en fait, et je ne peux pas me dissocier d'une partie ou d'une autre ", l'identité ethnique et l'appartenance civique à la société d'accueil sont complémentaires voire mutuellement enrichissantes. Dans ce sens, ce n'est pas l'identification ethnique mais plutôt le cumul de la racialisation sous forme d'une double discrimination, linguistique et raciale, pratiquée dans la société canadienne et dans la communauté francophone qui nuit au sentiment d'inclusion dans la francophonie et dans l'ensemble de la société canadienne. Il y a donc un besoin de renforcer davantage l'attachement des immigrés à la société d'accueil. (p. 189)

\section{...pour intégrer la communauté enseignante}

Dubar (2010) précise, à propos de l'étape de consolidation de l'expertise lors de laquelle la personne aspire à faire partie d'un corps professionnel, comme c'est le cas des NEll qui souhaitent intégrer l'équipe enseignante du milieu scolaire où ils évoluent, "que les professions forment des communautés unies autour des mêmes valeurs et de la même "éthique de service" [...] » (p. 131). Cavanagh, Cammarata et Blain (2016), pour leur part, rappellent que l'identité et la culture sont au centre du projet éducatif de l'école francophone en contexte minoritaire; nous avons $\mathrm{vu}$, à ce propos, que la diversification du corps enseignant imposait aux enseignants natifs des formes de négociations identitaires et interculturelles auxquelles ils avaient été, pour la plupart, peu exposés antérieurement. Afin de trouver la cohésion au sein de la communauté professionnelle francophone en milieu minoritaire, les fondements de cette identité et de cette culture doivent pourtant être partagés. Conséquemment, il y a lieu de croire que plus les valeurs, l'éthique et les savoirs des NEII qui entrent dans une école seront différents ou éloignés de ceux des enseignants déjà en place, plus leur intégration à cette communauté sera difficile. Si l'on y ajoute les marqueurs identitaires tels que l'origine ethnique, la religion, l'accent, les habitudes vestimentaires et alimentaires ou les conceptions antérieures du rôle de l'enseignant, l'on comprend que le processus d'acculturation, qui permettrait une intégration professionnelle et sociale complète du NEII, soit si complexe. Ainsi, la question de la profession vue comme une "communauté unie " autour de "valeurs ", d'une "éthique " et d'un "savoir " partagés nous apparaît comme l'élément clef du clivage "Eux/Nous" mis en lumière. En effet, la profession enseignante est structurée à partir de critères socioculturels définis historiquement par cette communauté et évolue au rythme des changements qui ont cours dans les sociétés desquelles elles font partie. Comme je l'ai exposé dans une publication précédente (Duchesne, 2017b), la plupart des NEII qui ont participé à mes travaux ont choisi l'assimilation comme stratégie d'intégration professionnelle afin de s'assurer d'obtenir le poste d'enseignant chèrement convoité ; ils ont ainsi, comme le font sans doute tous les nouveaux enseignants, mais en rencontrant plus d'obstacles que ces derniers, adopté de nouvelles pratiques pédagogiques et relationnelles, ou régulé celles existantes, afin de s'harmoniser aux valeurs, à l'éthique et aux savoirs préconisés dans leur nouvelle communauté professionnelle. 


\section{Conclusion}

Les NEII qui réussissent leur insertion en enseignement puis leur intégration à la profession enseignante dans les écoles de l'Ontario français manifestent de fortes aptitudes adaptatives. Ils adhèrent au rôle de passeur culturel qui leur est dévolu, autant du point de vue de la reproduction linguistique que de la promotion de la culture francophone, mais aussi de celle des différentes cultures, dont la leur, qui sont présentes au sein des écoles. Cet article a mis en lumière les enjeux de l'enseignement en milieu francophone minoritaire et les défis auxquels les nouveaux enseignants issus de l'immigration font face. Leur intégration à la communauté enseignante ne se fait pas sans difficulté en raison, notamment, des rivalités qui peuvent survenir lorsque les membres du groupe d'enseignants initialement en place ressentent leur sécurité linguistique de même que leur identité culturelle menacées par l'arrivée de nouveaux collègues qu'ils estiment appartenir à un groupe " autre » ou " différent » du leur. Les observations, constats et propos qui ont été rapportés dans cet article remettent en question les mécanismes d'accueil réservés aux enseignants d'immigration récente, mais également la préparation des enseignants natifs à accueillir ces nouveaux collègues. Bien que les efforts d'intégration reposent principalement sur les épaules des nouveaux arrivants, les communautés qui les accueillent ont la responsabilité de faciliter cette intégration en adoptant des perspectives plus inclusives de façon à réduire les impacts d'un potentiel clivage Eux/Nous au sein du corps professionnel.

\section{Références bibliographiques}

Balleux, A. et Perez-Roux, T. (2013). Transitions professionnelles. Recherche et formation, 74, $101-114$.

Cardinal, L. (1994). Ruptures et fragmentations de l'identité francophone en milieu minoritaire : un bilan critique. Sociologie et sociétés, 26(1), 71-86.

Cavanagh, M., Cammarata, L. et Blain, S. (2016) Enseigner en milieu francophone minoritaire canadien: synthèse des connaissances sur les défis et leurs implications pour la formation des enseignants. Revue canadienne de l'éducation, 39(4), 1-32.

Commission royale sur l'éducation. (1994). Pour l'amour d'apprendre : rapport de la Commission royale sur l'éducation. Ottawa : Gouvernement de l'Ontario. http://www.ontla.on.ca/library/repository/mon/25005/177143.pdf

Dubar, C. (2010). La socialisation (4 éd.). Paris : Armand Collin.

Duchesne, C. (2008). Difficultés d'insertion professionnelle en enseignement : le rôle des conceptions de l'éducation d'étudiants étrangers inscrits dans un programme de formation des maîtres en Ontario. Formation et pratiques d'enseignement en questions, 8, 119-139.

Duchesne, C. (2010a). À propos de l'accompagnement avant et pendant les stages d'étudiants immigrants inscrits à un programme de formation à l'enseignement. Revue des sciences de l'éducation, 36 (1), 95-115.

Duchesne, C. (2010b). L'apprentissage par transformation en contexte de formation professionnelle. Éducation et Francophonie, 38(1), 33-50.

Duchesne, C. (2017a). Enseignants issus de l'immigration et construction identitaire des élèves. Éducation Canada. https://www.edcan.ca/articles/enseignants-issus-de-limmigration-et-construction-identitaire-deseleves/?lang=fr

Duchesne, C. (2017b). Quelles stratégies d'acculturation des nouveaux enseignants issus de l'immigration privilégient-ils face aux défis culturels et identitaires de leur insertion professionnelle? Revue canadienne de l'éducation, 40(1), 1-24.

Duchesne, C. (soumis). Représentations racialisées d'enseignants issus de l'immigration dans les écoles de l’Ontario français. Revue des sciences de l'éducation de McGill.

Fourot, A.-C. (2016). Redessiner les espaces francophones au présent : la prise en compte de l'immigration dans la recherche sur les francophonies minoritaires au Canada. Politique et Sociétés, 35(1), 25-48. 
Gérin-Lajoie, D. (2006). Identité et travail enseignant dans les écoles de langue française situées en milieu minoritaire. Éducation et Francophonie, 34(1), 162-176.

Gérin-Lajoie, D. (2018). L'école de langue française et son rôle dans le développement du rapport à l'identité. Dans C. Isabelle (dir.) Système scolaire franco-ontarien : d'hier à aujourd'hui pour le plein potentiel des élèves (p. 41-57). Québec: Presses de l’Université du Québec.

Gouvernement du Canada. (2018). Les ministres responsables de l'immigration et de la francophonie canadienne annoncent un plan d'action conjoint visant à accroître l'immigration francophone. Ottawa : Immigration, Réfugiés et Citoyenneté Canada. https://www.canada.ca/fr/immigration-refugiescitoyennete/nouvelles/2018/03/les-ministres-responsables-de-limmigration-et-de-la-francophoniecanadienne-annoncent-un-plan-daction-conjoint-visant-a-accroitre-limmigra.html

Harvey, F. (1995). Le Québec et le Canada français : histoire d'une déchirure. Dans S. Langlois (dir.), Identité et cultures nationales. L'Amérique française en mutation (p. 49-64). Québec : Presses de l'Université Laval.

Huberman, M. (1989). La vie des enseignants. Neuchâtel, Suisse : Delachaux et Niestlé.

Jacquet, M., Moore, D. et Sabatier, C. (2008). Médiateurs culturels et insertion de nouveaux arrivants francophones africains : parcours de migration et perception des rôles. Glottopol, 11, 81-93.

Lalonde, A. (1995). Une mémoire de la nation. Les Fransaskois. Dans J. Mathieu (dir.) La mémoire dans la culture (p. 137-150). Québec: Presses de I’Université Laval.

Lefebvre, M. (2017, 2 novembre). L'enseignement, une carrière par défaut pour les immigrants francophones. Radio-Canada. https://ici.radio-canada.ca/nouvelle/1064298/immigrants-francophones-education-ontario

Létourneau, J. et Gani, R. (2017). S'intégrer à une nation passe-t-il par l’assimilation de son récit historique ? Réflexion à partir du cas québécois. Revue canadienne de l'éducation, 40(1), 1-27.

Lévesque, S., Croteau, J. et Gani, R. (2015). Conscience historique des jeunes francophones d'Ottawa : sentiment d'appartenance franco-ontarienne et récit du passé. Revue du Nouvel-Ontario, 40, 177-229.

Madibbo, A. (2009-2010). Pratiques identitaires et racialisation des immigrants africains francophones en Alberta. Canadian Ethnic Studies/Études ethniques au Canada, 40-41(3-1), 175-189.

Ministère de l'Éducation de l'Ontario. (2011). Monographie $n^{\circ} 10$ : Intégration de la culture dans les pratiques pédagogiques. Centre franco-ontarien de ressources pédagogiques. http://www.edu.gov.on.ca/fre/teachers/studentsuccess/monographie10.pdf

Ministère de l'Éducation de l'Ontario. (2014). Consultation sur l'aménagement linguistique dans les écoles de langue française en Ontario : rapport de consultation. http://ressources.cforp.ca/fichiers/documentspal/pal-rapport-consultation-vf.pdf

Morrissette, J. et Demazière, D. (2018). Dualité des processus de socialisation professionnelle des enseignants formés hors Québec : entre imposition et appropriation. Alterstice, 8(1), 95-106.

Morrissette, J., Charara, Y., Boily, A., et Diédhiou, B. (2016). Les stratégies des accompagnateurs de l'intégration socioprofessionnelle des enseignants formés à l'étranger : le jeito des despachantes. Revue canadienne de l'éducation, 39(1), 1-29.

Mulatris, P. (2009). Francophonie albertaine et inclusion des nouveaux arrivants : post mortem à un débat sur un changement de nom. International Migration and Integration, 10, 145-158.

Mulatris, P. et Skogen, R. (2012). Pour une inclusion complète : I'insertion professionnelle des étudiants stagiaires des minorités visibles dans l'école francophone albertaine. Revue internationale d'études canadiennes, 4546, 331-352.

Murphy, M. (2018). Le Canada à la recherche de profs francophones. L'Express-emploi, 10 septembre. https://www.lexpress.fr/emploi/gestion-carriere/le-canada-a-la-recherche-de-profsfrancophones_2032569.html 
Niyubahwe, A., Mukamurera, J. et Jutras, F. (2013). Professional integration of immigrant teachers in the school system: A literature review. Revue des sciences de l'éducation de McGill, 48(2), 279-296.

Niyubahwe, A., Mukamurera, J. et Jutras, F. (2014). L'expérience de transition professionnelle des enseignants de migration récente au Québec. Revue canadienne de l'éducation, 37(4), 1-32.

Ordre des enseignantes et des enseignants de l'Ontario (OEEO). (2017). Transition à l'enseignement. https://www.oeeo.ca//media/PDF/2017\%20Transition\%20to\%20Teaching/2017\%20T2T\%20Main\%20Report $\% 20 F r \_F i n a l . p d f$

Pierroz, S. (2018). Pénurie des enseignants francophones, un problème toujours irrésolu. \#ONfr - TFO, 6 septembre. https://onfr.tfo.org/penurie-des-enseignants-francophones-un-probleme-toujours-irresolu/

Sinacore, A. L., Park-Saltzman, J., Mikhail, A. M. et Wada, K. (2010). Falling through the cracks: Academic and career challenges faced by immigrant graduate students. Canadian Journal of Counselling and Psychotherapy, 45(2), 168-187.

Statistique Canada. (2011). Le français et la francophonie au Canada ( $n^{\circ}$ 98-314-X2011003 au catalogue). https://www12.statcan.gc.ca/census-recensement/2011/as-sa/98-314-x/98-314-x2011003_1-fra.pdf

Sylvestre, P. F. (2013). L'Ontario français, quatre siècles d'histoire. Ottawa: Éditions David.

Sylvestre, P. F. et Lévesque, S. (2018). Historique des écoles de langue française en Ontario. Dans C. Isabelle (dir.), Système scolaire franco-ontarien : d'hier à aujourd'hui pour le plein potentiel des élèves (p. 7-39). Québec: Presses de l'Université du Québec. 